\title{
Topiramate in the treatment of Prader-Willi syndrome: A case report
}

\author{
Nicole East, PharmD ${ }^{1}$ \\ Megan Maroney, PharmD, BCPP²
}

How to cite: East N, Maroney M. Topiramate in the treatment of Prader-Willi syndrome: A case report. Ment Health Clin [Internet]. 2017;7(1):7-9. DOI: 10.9740/ mhc.2017.01.007.

\begin{abstract}
Background: Prader-Willi syndrome (PWS), a neurologic disorder caused by a mutation of chromosome 15, is characterized by such symptoms as hypotonia, hypogonadism, hyperphagia, cognitive impairment, and difficult behaviors. One of the most concerning symptoms is hyperphagia, which can lead to uncontrolled obesity. Obesity is a major cause of increased morbidity and mortality in patients with PWS; however, diagnosing PWS early in life improves the prognosis.

Case Summary: An 11-year-old African American boy with a past medical history significant for PWS, attention deficit/hyperactivity disorder, oppositional defiant disorder, obesity, and asthma was admitted after he became violent and destructive at his foster home while trying to get food. The patient had a 28 day stay on the children's crisis intervention unit where quetiapine was discontinued and he was maintained on clonidine $0.1 \mathrm{mg} 3$ times daily, hydroxyzine $25 \mathrm{mg}$ in the morning and $50 \mathrm{mg}$ at bedtime, montelukast 5 $\mathrm{mg}$ daily and titrated on methylphenidate $10 \mathrm{mg}$ in the morning and $5 \mathrm{mg}$ in the afternoon, topiramate 100 $\mathrm{mg}$ twice daily, and aripiprazole $10 \mathrm{mg}$ twice daily. The patient displayed improved behavior control and less food-related aggression; he denied any side effects of medications.

Discussion: This case demonstrates the positive effects of topiramate for reducing aggression and demand for food in a child with PWS most likely due to an increase in satiety. It is hard to definitively attribute the positive response directly to topiramate. Further research should be conducted to determine if topiramate is an effective treatment option in these individuals.
\end{abstract}

Keywords: topiramate, Prader-Willi syndrome, youth

\begin{abstract}
${ }^{1}$ (Corresponding author) Clinical Assistant Professor of Pharmacy Practice, Fairleigh Dickinson, University School of Pharmacy, Florham Park, New Jersey; Clinical Pharmacist-Internal Medicine, Community Medical Center, Toms River, New Jersey, neast@fdu.edu, ORCID: http:// orcid.org/0000-0002-6038-9404; ${ }^{2}$ Clinical Assistant Professor, Ernest Mario School of Pharmacy, Rutgers State University of New Jersey, New Brunswick, New Jersey; Clinical Psychiatric Pharmacist, Monmouth Medical Center, Long Branch, New Jersey, ORCID: http://orcid.org/oooo$0002-2718-6787$
\end{abstract}

Disclosure: The authors have no potential conflicts of interest related to this manuscript.

\section{Background}

Prader-Willi syndrome (PWS) is a neurologic disorder caused by a mutation of chromosome 15 . Prader-Willi syndrome can be a result of 3 main genetic errors: noninherited deletion in the paternally contributed chromosome 15, maternal uniparental disomy, or an error in the imprinting process. It is characterized by hypotonia, hypogonadism, hyperphagia, cognitive impairment, and difficult behaviors, including mood disturbances, selfabusive behavior, and compulsive-eating disorder. These types of behaviors can lead to increased morbidity and mortality and can have detrimental effects on a patient's mental health.

Hyperphagia is often the most concerning issue in these patients due to increased risk for obesity and other cardiometabolic complications. When diagnosed early, PWS prognosis improves because patients are better able 
to control diet and weight. ${ }^{1}$ Patients and families can attempt behavioral modifications for weight loss, including decreasing caloric intake and following exercise programs; however, poor adherence may be a barrier to success due to the long-term commitment needed. ${ }^{2}$ There is an unmet need for targeted pharmacotherapy for hyperphagia in this patient population. Currently, there is no US Food and Drug Administration-approved medication or surgical intervention indicated for this or any symptom associated with PWS. ${ }^{1}$

Here, we present the case of an 11-year-old male patient with PWS who demonstrated severely violent behaviors secondary to compulsive-eating disorder, resulting in admission to an inpatient psychiatric facility. The use of topiramate, an anticonvulsant with the side effect of appetite suppression, resulted in dramatic improvement in this patient. ${ }^{3}$

\section{Case Report}

The patient was an 11-year-old African American boy with a past medical history significant for PWS, attention deficit/hyperactivity disorder, oppositional defiant disorder, obesity and asthma. Per report, the patient became violent and destructive at his foster home when he was denied excessive amounts of food. His home medications included clonidine $0.1 \mathrm{mg} 3$ times daily for attention deficit/hyperactivity disorder, hydroxyzine $25 \mathrm{mg}$ daily for anxiety and $50 \mathrm{mg}$ at bedtime for anxiety and insomnia, montelukast $5 \mathrm{mg}$ at bedtime for asthma, and quetiapine $100 \mathrm{mg}$ twice daily for mood stabilization. All home medications were continued upon admission to the children's crisis unit. Per the foster mother, in the past, when quetiapine was introduced, the patient's compulsive-eating and violent behaviors toward wanting food worsened. The patient had been given benztropine and aripiprazole in the past but their effects were undocumented and no other psychiatric history was known. Per the foster mother, the biological father had a diagnosis of schizophrenia, and there was a history of anxiety on the maternal side.

On days 2 and 3 of admission, the patient was eating excessively, was demanding food, and required constant redirection. At this time, quetiapine was discontinued and aripiprazole $5 \mathrm{mg}$ daily, methylphenidate $5 \mathrm{mg}$ daily, and topiramate $25 \mathrm{mg}$ twice daily were added to the treatment regimen. The patient responded well to the medication in terms of aggression by day 4. On day 6 , the patient attacked staff and continued to be aggressive about food and resistant to healthy choices offered to him. At this time, topiramate was increased to $50 \mathrm{mg}$ twice daily. Additionally, methylphenidate was increased to $10 \mathrm{mg}$ daily. The patient had to be placed in restraints for 2 hours on day 8 due to violent and threatening behaviors unrelated to food. After the incident, aripiprazole was increased to $10 \mathrm{mg}$ twice daily, topiramate was increased to $100 \mathrm{mg}$ twice daily, and both divalproex 250 $\mathrm{mg}$ twice daily and methylphenidate $5 \mathrm{mg}$ before lunch were added. On day 10, the patient was still seeking food but behaviors remained in control. On days 11 through 14 , the patient was able to control impulses when told to wait for food and denied any side effects of medications. The patient was ready for discharge on day 15 ; however, he had to wait for appropriate postdischarge placement. On days 16 through 28, the patient displayed improved behavior control, had less food-related aggression, and was verbal and helpful on the unit; the patient acknowledged that he was "happy on his medications" and "doesn't eat as much" due to his lessened appetite. The patient was discharged on day 28 on the following medications: clonidine $0.1 \mathrm{mg} 3$ times daily, hydroxyzine $25 \mathrm{mg}$ in the morning and $50 \mathrm{mg}$ at bedtime, montelukast $5 \mathrm{mg}$ at bedtime, methylphenidate $10 \mathrm{mg}$ in the morning and $5 \mathrm{mg}$ in the afternoon, topiramate $100 \mathrm{mg}$ twice daily, and aripiprazole $10 \mathrm{mg}$ twice daily.

\section{Discussion and Conclusion}

Studies of various psychopharmacologic interventions have demonstrated positive effects on self-injurious behavior, but few have shown improvements in compulsive eating and weight. ${ }^{4,5}$ A PubMed search revealed 2 open-label trials, 1 in adults and 1 in a youth population, which analyzed the effectiveness of topiramate in PWS, and 1 case series of 3 adult case reports that looked at attenuation of self-injurious behaviors. ${ }^{2,6,7}$ Two of these studies examined effects on appetite and weight specifically. ${ }^{2,6}$ In all the trials, each person had a confirmed diagnosis of PWS and was titrated to an effective dose of topiramate. ${ }^{2,6,7}$

Smathers et $\mathrm{al}^{2}$ described an open-label trial of 8 patients between the ages of 10 and 19 years who were titrated to a dose of 100 to $600 \mathrm{mg} / \mathrm{d}$ of topiramate. Patients in this study had undergone a trial of behavioral, dietary, and exercise regimens before initiating topiramate. No changes to the patients' medications or regular routine were allowed during the study period. All 7 patients who completed this study either lost weight or showed a reduced rate of weight gain compared with before initiating topiramate. Parents also noted a decrease in compulsive-eating behaviors. Additional improvements included decreased aggression and improvements in mood in all 7 study completers as well as reduced selfabusive behavior in 2 patients. ${ }^{2}$

Another open-label study was conducted by Shapira and colleagues $^{6,7}$ in 8 adult patients with PWS who ranged in 
age from 19 to 38 years. This 8-week trial assessed appetite, weight, and behaviors, including self-injury. Topiramate was titrated to an average dose of $162.5 \mathrm{mg}$ at bedtime. It was generally well tolerated but did not decrease body mass index, appetite, or caloric intake during periods of free access to food. Topiramate did, however, demonstrate improvements in self-injurious behavior in this patient population. ${ }^{6,7}$

The current case report demonstrates the positive effects of topiramate for reducing aggression and demand for food, most likely due to an increase in satiety. Though it is hard to definitively attribute the positive response directly to topiramate, it is possible that the significant improvement in this child's aggressive behaviors toward food was reduced due to the known side effect of decrease in appetite with this medication. ${ }^{3}$ The results of this case study are similar to the results of the previously described youth trial that found a positive effect on negative behaviors and compulsive eating in children and adolescents with PWS treated with topiramate. ${ }^{2}$ These results are also consistent with the data presented in a recent review of the published literature on psychotropic treatment options in patients with PWS. ${ }^{8}$

The addition of methylphenidate may also have contributed to this patient's behavior and appetite improvements; however, central nervous system stimulants have not previously shown benefit in patients with PWS. ${ }^{5}$ The discontinuation of quetiapine may also have improved the patient's appetite and food-related behaviors, though the patient still demonstrated similar food-related aggression throughout his stay after quetiapine was discontinued. In addition to the quetiapine, at admission the patient was taking hydroxyzine, an antihistamine, which could have contributed to his aggression about food due to the proposed side effect of appetite stimulation. ${ }^{9}$ Though the results cannot be directly attributed to the topiramate because methylphenidate was added and quetiapine was discontinued at the same time topiramate was initiated, this case illustrates topiramate's effectiveness as part of a comprehensive treatment plan.

Positive health outcomes have been shown to be associated with early weight control in patients with PWS. ${ }^{1}$ Based on 1 small trial and this case report, topiramate seems to be a promising treatment option for young patients with PWS. Since medication options in this disease are limited, further research should be conducted to determine if topiramate is effective in these individuals.

\section{References}

1. Prader-Willi Syndrome Association [Internet]. Chicago: The Association; C2004-15 [updated 2015; cited 2014 Dec 29]. Medical Overview of PWS. Available from: http://pwsausa.org/physiciansresources/.

2. Smathers SA, Wilson JG, Nigro MA. Topiramate effectiveness in Prader-Willi syndrome. Pediatr Neurol. 2003;28(2):130-3. PubMed PMID: 12699864

3. Topiramate [package insert]. Titusville (NJ): Janssen Pharmaceuticals, Inc; 2014.

4. Yaryura-Tobias JA, Grunes MS, Bayles ME, Neziroglu F. Hyperphagia and self-mutilation in Prader-Willi syndrome: psychopharmacological issues. Eat Weight Disord. 1998;3(4):163-7. PubMed PMID: 10728166.

5. Dykens E, Shah B. Psychiatric disorders in Prader-Willi syndrome: epidemiology and management. CNS Drugs. 2003;17(3):167-78. PubMed PMID: 12617696.

6. Shapira NA, Lessig MC, Lewis MH, Goodman WK, Driscoll DJ. Effects of topiramate in adults with Prader-Willi syndrome. Am J Ment Retard. 2004;109(4):301-9. DOI: 10.1352/0895$8017(2004) 109<301$ : EOTIAW>2.0.CO;2. PubMed PMID:15176917.

7. Shapira NA, Lessig MC, Murphy TK, Driscoll DJ, Goodman WK. Topiramate attenuates self-injurious behaviour in Prader-Willi Syndrome. Int J Neuropsychopharmacol. 2002;5(2):141-5. DOI: 10. 1017/S1461145702002833. PubMed PMID:12135538.

8. Bonnot O, Cohen D, Thuilleaux D, Consoli A, Cabal S, Tauber M. Psychotropic treatments in Prader-Willi syndrome: a critical review of published literature. Eur J Pediatr. 2016;175(1):9-18. DOI: 10.1007/s00431-015-2670-x. PubMed PMID:26584571.

9. Jørgensen EA, Knigge U, Warberg J, Kjaer A. Histamine and the regulation of body weight. Neuroendocrinology. 2007;86(3):2104. DOI: 10.1159/000108341. PubMed PMID: 17848791. 